\title{
Clinical Evaluation of a New Rapid Immunochromatographic Test for Detection of Bordetella Pertussis Antigen
}

\section{Kenji Okada}

Division of Basic Nursing, Fukuoka Nursing College

\section{Yuho Horikoshi}

Division of Infectious Diseases, Department of Pediatrics, Tokyo Metropolitan Children's Medical Center

\section{Naoko Nishimura}

Department of Pediatrics, Konan Kosei Hospital

\section{Shigeki Ishii}

Department of Pediatrics, Miyazaki Prefectural Miyazaki Hospital

\section{Hiroko Nogami}

Department of Respiratory Medicine, National Hospital Organization Fukuoka National Hospital

\section{Chikako Motomura}

Department of Pediatrics, National Hospital Organization Fukuoka National Hospital

\section{Isao Miyairi}

Division of Infectious Diseases, Department of Medical Subspecialties, National Center for Child Health and Development

\section{Naoki Tsumura}

Tsumura Family Clinic

\section{Toshihiko Mori}

Department of Pediatrics, NTT East Sapporo Hospital

\section{Kenta Ito}

Department of General Pediatrics, Aichi Children's Health and Medical Center

\section{Shinichi Honma}

Honma Children's Clinic

\section{Kensuke Nagai}

Nagai Pediatric Clinic

\section{Hiroshi Tanaka}

Sapporo Cough Asthma and Allergy Center

Toru Hayakawa ( $\nabla$ hayakawa.th@om.asahi-kasei.co.jp )

Asahi Kasei (Japan)

\section{Chiharu Abe}

\section{Asahi Kasei (Japan)}


Kazunobu Ouchi

Department of Medical Welfare for Children, Kawasaki University of Medical Welfare

\section{Research Article}

Keywords: Bordetella pertussis, immunochromatographic antigen kit (ICkit), real-time polymerase chain reaction ( $\mathrm{PPCR}$ ).

Posted Date: September 24th, 2021

DOl: https://doi.org/10.21203/rs.3.rs-902448/v1

License: (c) (i) This work is licensed under a Creative Commons Attribution 4.0 International License. Read Full License 


\section{Abstract}

A more rapid and less complicated test is required in clinical settings to diagnose pertussis. We need to detect Bordetella pertussis, which mainly causes pertussis, as early as possible, because pertussis is more likely to become severe in infants, and people around them can easily become the source of infection due to its strong infectivity. Nevertheless, methods that can detect $B$. pertussis rapidly and efficiently are lacking. Therefore, we developed a new immunochromatographic antigen kit (ICkit) for the early diagnosis of pertussis. The ICkit detects $B$. pertussis antigens in a nasopharyngeal swab without equipment and provides the result in about 15 min with a simple procedure.

Additionally, a prospective study to evaluate the ICkit was conducted in 11 medical institutions, involving 195 cases with suspected pertussis. The sensitivity and specificity of the ICkit were $86.4 \%(19 / 22)$ and $97.1 \%(168 / 173)$, respectively, compared with the real-time polymerase chain reaction (rPCR). The ICkit detected the antigen in both children and adults. Furthermore, the ICkit detected the antigen until the 25th day from the onset of cough, when rPCR detected the antigen. Thus, the ICkit demonstrated a high correlation with rPCR and would help diagnose pertussis more rapidly and efficiently.

\section{Introduction}

Pertussis, known as whooping cough, is a highly contagious respiratory infection mainly caused by Bordetella pertussis. B. pertussis spreads easily from person to person due to its strong infectivity [1]. Cases of pertussis are still frequently reported worldwide [2].

When $B$. pertussis infects unvaccinated infants, the disease can be severe and may result in death $[3,4]$. Globally, it was estimated that there were about 24.1 million infected cases and 160,700 deaths due to pertussis in children under the age of five years in 2014 [5]. In addition, the presence of infected adults around unvaccinated infants poses a risk of transmission to the infants [6,7]. Although pertussis is a vaccine-preventable disease, it has recently been reported that infection spreads among adults whose vaccine efficacy has diminished over time [8].

Pertussis has non-specific symptoms such as rhinorrhea, sneezing, and non-specific cough during the catarrhal phase, and characteristic symptoms such as paroxysms, whooping, post-tussive vomiting, and apnea during the paroxysmal phase $[9,10]$. The severity and characteristics of the symptoms vary depending on the age of the patient, duration of infection, and vaccination [11, 12]. Since there are often no specific clinical symptoms, it is difficult to diagnose based on clinical symptoms alone [13]. Adenoviruses, respiratory syncytial viruses (RSV), human parainfluenza viruses, influenza viruses, Haemophilus influenzae, Mycoplasma pneumoniae, and other agents may also cause pertussis-like symptoms [14-16]. Infants hospitalized with acute respiratory tract infections may have pertussis without typical symptoms $[17,18]$. Thus, diagnosis based on laboratory confirmation is essential, along with clinical symptoms. 
There are cultures, nucleic acid tests, and serological tests for the diagnosis of pertussis $[9,19]$. Of these tests, the direct tests such as cultures and nucleic acid tests help determine infectivity and the need for antimicrobials, unlike the indirect tests such as antibody tests. However, the existing direct tests are often time-consuming and limited in use due to facility conditions, so that the requirements for the direct tests have not yet been fully met. Therefore, we often have difficulties in getting the test results at the patient's first visit. Especially in the clinic that patients visit initially, it is challenging to perform the tests due to a shortage of equipment and technical personnel. Thus, a novel direct test is required to enable rapid and straightforward detection without equipment.

A new rapid immunochromatographic antigen kit (ICkit) that is simple for all users, can be used for pointof-care testing (POCT), is a standalone test without requiring any equipment, and provides rapid results with a decision time of 15 min was developed. Figure 1 shows the structure and principle of the ICkit. The ICkit targets the ribosomal protein L7/L12 antigen, which is a component of the 50 S ribosome and contains an amino acid sequence specific to each bacterial species [20-23]. Sano et al. and lto et al. reported the ICkit's utility in detecting the ribosomal protein L7/L12 in diagnosing Mycoplasma pneumonia and Legionella pneumonia [24, 25]. Similarly, the ICkit's usefulness for detecting $B$. pertussis is confirmed in the present clinical study.

\section{Results}

\section{Dilution sensitivity test.}

Table 1 shows the result of the dilution sensitivity test. The ICkit was positive with $2.2 \times 10^{4} \mathrm{CFU} / \mathrm{mL}$ and more for all strains (BAA-589, 8467, 9797, and 9340). Therefore, the detection limit of the ICkit was estimated to be $2.2 \times 10^{4} \mathrm{CFU} / \mathrm{mL}$. 
Table 1

Dilution sensitivity test with the ICkit for four different strains of Bordetella pertussis.

\begin{tabular}{|c|c|c|}
\hline $\begin{array}{l}\text { Strain } \\
\text { (ATCC\#) }\end{array}$ & $\begin{array}{l}\text { Concentration } \\
(\mathrm{CFU} / \mathrm{mL})\end{array}$ & ICkit Result \\
\hline \multirow[t]{4}{*}{ BAA-589 } & $44 \cdot 10^{4}$ & + \\
\hline & $8.8 \cdot 10^{4}$ & + \\
\hline & $2.2 \cdot 10^{4}$ & + \\
\hline & $0.4 \cdot 10^{4}$ & - \\
\hline \multirow[t]{4}{*}{8467} & $44 \cdot 10^{4}$ & + \\
\hline & $8.8 \cdot 10^{4}$ & + \\
\hline & $2.2 \cdot 10^{4}$ & + \\
\hline & $0.4 \cdot 10^{4}$ & - \\
\hline \multirow[t]{4}{*}{9797} & $44 \cdot 10^{4}$ & + \\
\hline & $8.8 \cdot 10^{4}$ & + \\
\hline & $2.2 \cdot 10^{4}$ & + \\
\hline & $0.4 \cdot 10^{4}$ & - \\
\hline \multirow[t]{4}{*}{9340} & $44 \cdot 10^{4}$ & + \\
\hline & $8.8 \cdot 10^{4}$ & + \\
\hline & $2.2 \cdot 10^{4}$ & + \\
\hline & $0.4 \cdot 10^{4}$ & - \\
\hline
\end{tabular}

\section{Cross-reactivity test.}

The cross-reactivity test identified cross-reactivity of the ICkit with B. parapertussis and B. holmesii. However, it identified no cross-reactivity with the other pathogens in the ICkit (Supplementary Table S2).

Clinical study. 
Written, informed consent was obtained from a total of 203 cases, and they were enrolled in this study. Finally, 195 cases, excluding eight cases, were analyzed. The eight cases were excluded because it was found after the study that they did not meet the inclusion criteria or did not get any ICkit results.

Table 2 and Table 3 show baseline demographics and clinical symptoms by age. There were 151 cases $(77.4 \%)$ aged 14 years or younger and 44 cases $(22.6 \%)$ aged 15 years or older. The median age was three years (range: 0 to 69 years). At baseline, the group aged 14 years or younger had a higher proportion of males, inpatients, vaccination, and antimicrobials before testing than the group aged 15 years or older. Both groups had contacts with infected cases at a rate of about $30 \%$. All cases except one had a cough, whereas 54 cases $(27.7 \%)$ had a fever. The median duration of cough was 13 days (range: 2 to 149 days). The group aged 14 years and younger had a higher incidence of fever, whooping, and apnea than those aged 15 years and older. Both groups had a similar rate of paroxysms and post-tussive vomiting.

Table 2

Baseline demographics.

\begin{tabular}{|c|c|c|c|c|c|}
\hline \multirow[b]{2}{*}{ Sex } & \multirow[b]{2}{*}{ Male } & \multicolumn{2}{|c|}{$\begin{array}{l}14 \text { years or younger ( } 151 \\
\text { cases) }\end{array}$} & \multicolumn{2}{|c|}{$\begin{array}{l}15 \text { years or older ( } 44 \\
\text { cases) }\end{array}$} \\
\hline & & 85 & $56.3 \%$ & 11 & $25.0 \%$ \\
\hline & Female & 66 & $43.7 \%$ & 33 & $75.0 \%$ \\
\hline \multirow[t]{2}{*}{ Severity } & Outpatient & 96 & $63.6 \%$ & 43 & $97.7 \%$ \\
\hline & Inpatient & 55 & $36.4 \%$ & 1 & $2.3 \%$ \\
\hline \multirow[t]{3}{*}{ Vaccination } & Present & 92 & $60.9 \%$ & 13 & $29.5 \%$ \\
\hline & Absent & 49 & $32.5 \%$ & 0 & $0.0 \%$ \\
\hline & Unknown & 10 & $6.6 \%$ & 31 & $70.5 \%$ \\
\hline \multirow{3}{*}{$\begin{array}{l}\text { Antimicrobials } \\
\text { before testing }\end{array}$} & Present & 46 & $30.5 \%$ & 7 & $15.9 \%$ \\
\hline & Absent & 104 & $68.9 \%$ & 34 & $77.3 \%$ \\
\hline & Unknown & 1 & $0.6 \%$ & 3 & $6.8 \%$ \\
\hline \multirow{2}{*}{$\begin{array}{l}\text { Contact with infected } \\
\text { cases }\end{array}$} & Present & 57 & $37.7 \%$ & 15 & $34.1 \%$ \\
\hline & Absent & 94 & $62.3 \%$ & 29 & $65.9 \%$ \\
\hline
\end{tabular}


Table 3

Clinical symptoms.

\begin{tabular}{|c|c|c|c|c|c|}
\hline \multirow[b]{2}{*}{ Fever } & \multirow[b]{2}{*}{ positive } & \multicolumn{2}{|c|}{$\begin{array}{l}14 \text { years or younger } \\
\text { (151 cases) }\end{array}$} & \multicolumn{2}{|c|}{$\begin{array}{l}15 \text { years or older } \\
\text { (44 cases) }\end{array}$} \\
\hline & & 49 & $32.5 \%$ & 5 & $11.4 \%$ \\
\hline & negative & 102 & $67.5 \%$ & 39 & $88.6 \%$ \\
\hline \multirow[t]{2}{*}{ Cough } & positive & 150 & $99.3 \%$ & 44 & $100.0 \%$ \\
\hline & negative & 1 & $0.7 \%$ & 0 & $0.0 \%$ \\
\hline \multirow[t]{4}{*}{ Duration of cough (days) } & $<15$ & 79 & $52.3 \%$ & 22 & $50.0 \%$ \\
\hline & 15 to 28 & 45 & $29.8 \%$ & 15 & $34.1 \%$ \\
\hline & $>28$ & 26 & $17.2 \%$ & 7 & $15.9 \%$ \\
\hline & none & 1 & $0.7 \%$ & 0 & $0.0 \%$ \\
\hline \multirow[t]{2}{*}{ Paroxysms } & positive & 119 & $78.8 \%$ & 36 & $81.8 \%$ \\
\hline & negative & 32 & $21.2 \%$ & 8 & $18.2 \%$ \\
\hline \multirow[t]{2}{*}{ Whooping } & positive & 27 & $17.9 \%$ & 2 & $4.5 \%$ \\
\hline & negative & 124 & $82.1 \%$ & 42 & $95.5 \%$ \\
\hline \multirow[t]{2}{*}{ Post-tussive vomiting } & positive & 57 & $37.7 \%$ & 15 & $34.1 \%$ \\
\hline & negative & 94 & $62.3 \%$ & 29 & $65.9 \%$ \\
\hline \multirow[t]{2}{*}{ Apneas } & positive & 31 & $20.5 \%$ & 0 & $0.0 \%$ \\
\hline & negative & 120 & $79.5 \%$ & 44 & $100.0 \%$ \\
\hline
\end{tabular}

Table 4 summarizes the correlations of the ICkit and culture results with real-time PCR (rPCR) results. The sensitivity and the specificity of the ICkit were $86.4 \%(19 / 22,95 \% \mathrm{Cl}$ : 66.7 to $95.3 \%)$ and $97.1 \%(168 / 173$, 95\%Cl: 93.4 to $98.8 \%)$ compared with rPCR, and those of culture were $63.6 \%(14 / 22,95 \% \mathrm{Cl}: 43.0$ to $80.3 \%$ ) and $97.6 \%$ (160/164, 95\% Cl: 93.9 to $99.0 \%)$ compared with rPCR, respectively. The sensitivity and the specificity of the ICkit were $72.2 \%(13 / 18,95 \% \mathrm{Cl}: 49.1$ to $87.5 \%)$ and $94.0 \%(158 / 168,95 \% \mathrm{Cl}$ : 89.4 to $96.7 \%)$, respectively, compared with the culture (Supplementary Table S1). 
Table 4

Sensitivity, specificity, and concordance rate of the ICkit and culture compared with rPCR.

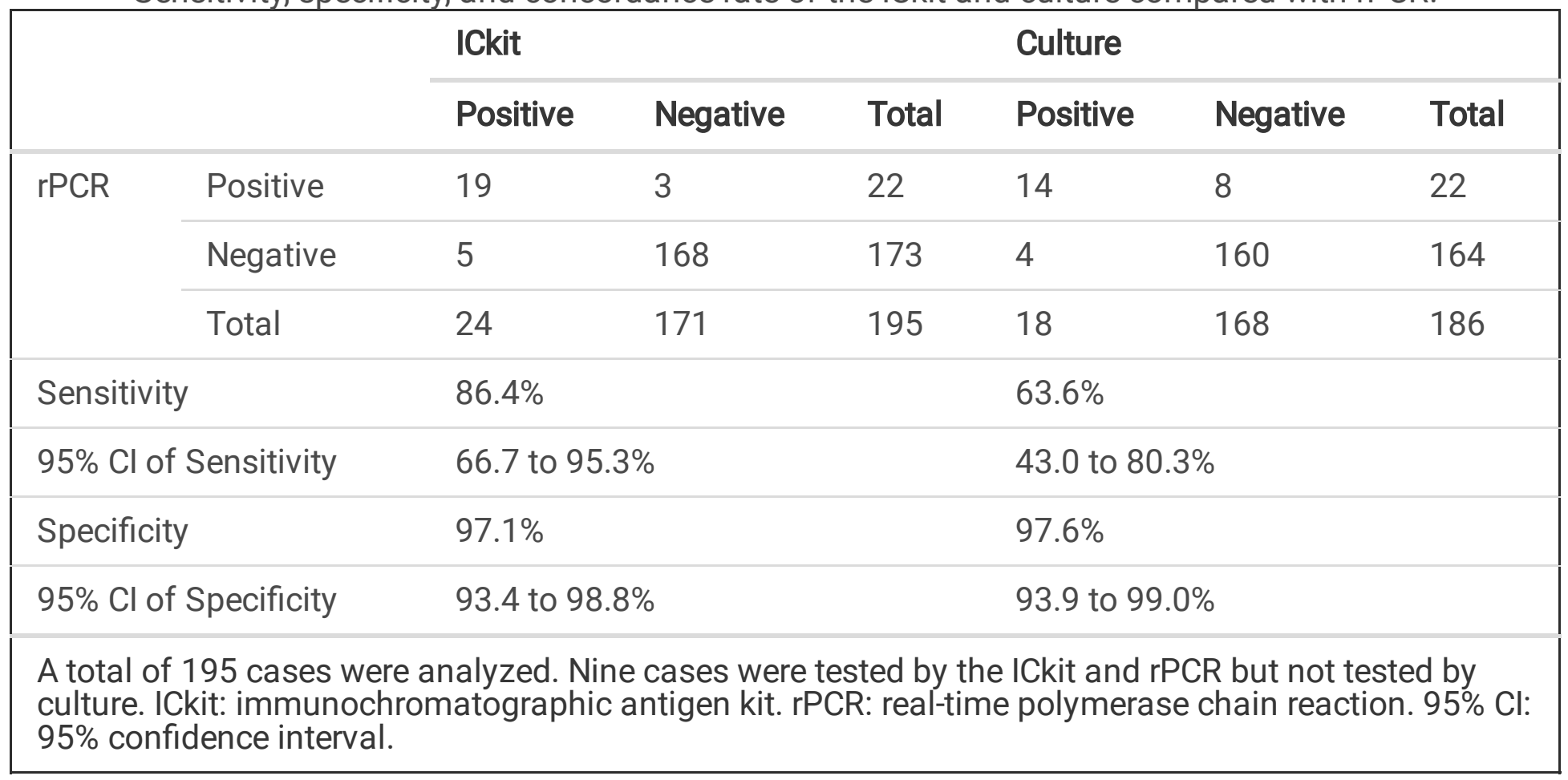

Figure 2 shows the relationship between the results of the ICkit and the number of IS 481 genes. There were many positive cases on rPCR from $1.0 \times 10^{5}$ to $1.0 \times 10^{7}$ copies/swab. The ICkit was negative in order from the group with the fewest copies in a swab. The ICkit was negative in two cases with $1.0 \times 10^{4}$ copies/swab and in one case with $1.0 \times 10^{5} \mathrm{copies} / \mathrm{swab}$. Therefore, the detection limit of the ICkit was estimated to be around $1.0 \times 10^{5}$ copies/swab.

Figure 3 shows the relationship between test results and age. Both groups aged 14 years and younger and 15 years or older had positive cases with the ICkit, similar to rPCR. However, each test's proportion of positive cases was higher in the group aged 14 years and younger than in those aged over 15 years or older. Figure 4 shows the relationship between test results and infection duration. Infection duration was defined as the number of days from cough onset to testing. The number of positive cases with the ICkit was almost the same as the number of positive cases with rPCR for each duration. None of the tests had positive cases in the group longer than 28 days. The ICkit and rPCR provided positive results up to day 25 , and culture provided positive results up to day 20 .

\section{Discussion}

Pertussis is a highly contagious infectious disease that is still widespread in both children and adults. The problem with pertussis is that unvaccinated infants are at risk of severe illness and death. Moreover, people around the infants can be the source of infection due to the decreased effectiveness of the vaccine. Nevertheless, no tests can detect the infection early and efficiently enough to treat or prevent the disease [12]. All existing tests require specialized equipment and trained personnel, which makes it difficult to access the tests. In addition, pertussis is highly infectious, so it is essential to have a test that 
provides rapid results at the patient's first visit. We need to improve the inadequate access to testing for infected people. Especially in primary care facilities that most infected people visit first, it takes time to obtain test results due to the lack of testing capability.

We developed an immunochromatographic antigen test to improve access to testing. The ICkit result can be checked in $15 \mathrm{~min}$ after dropping the sample without specialized equipment. The dilution sensitivity test confirmed that the ICkit could detect the antigens of $B$. pertussis strains. Furthermore, this was the first prospective clinical study using the ICkit for the diagnosis of pertussis. The usefulness of the ICkit was evaluated in 195 cases suspected of being infected with B. pertussis. Their baseline demographics and clinical symptoms (Table 2 and Table 3 ) were similar to those previously reported [1]. There were many inpatient cases in infants without vaccination, while there were many mild cases in adults. Antimicrobial treatment started before confirming the laboratory results due to its seriousness when inpatients and infants had probable pertussis. Several people were enrolled in the study after contact with people suspected of having pertussis. Almost all cases had a cough, but fewer cases with probable pertussis had a fever than with other infections. The duration of the cough was less than 28 days in most cases. For specific symptoms, paroxysms and post-tussive vomiting were common in children and adults. On the other hand, whooping and apnea episodes were seen in children, but rarely in adults.

The clinical performance of the ICkit was well correlated with its sensitivity of $86.4 \%(19 / 22)$ and specificity of $97.1 \%$ (168/173) compared to rPCR. The ICkit's specificity compared to culture was high, but its sensitivity compared to culture was lower than that compared to rPCR (Supplementary Table S1). When comparing the ICkit and culture to rPCR, the sensitivity of the ICkit was higher than that of culture (Table 4). Since rPCR is the most sensitive of pertussis tests [26], the sensitivity of the ICkit, which correlated well with the sensitivity of rPCR, is considered to be higher than that of culture.

There were eight cases in which the results of rPCR and the ICkit did not match. In the three cases that were rPCR-positive and ICkit-negative, it was suspected that the results of the ICkit were false-negative due to an antigen amount below the detection limit of the ICkit, because the three cases had a smaller number of IS 481 genes in the swabs, as shown in Fig. 2. There is a similar report on the relationship between quantitative rPCR and culture [27]. This result showed that it is crucial to secure enough antigen by proper sample collection to obtain optimal results with the ICkit. The CDC describes a precise method for collecting a nasopharyngeal (NP) swab that applies to direct tests for B. pertussis [28]. The doctors' diagnoses also supported positives for pertussis in the three cases. In the five cases that were rPCRnegative and ICkit-positive, one of them seemed to be a false-negative rPCR result based on the doctor's diagnosis. The other four cases were presumed to be false-positive results of the ICkit. False-positive results for immunochromatographic antigen kits have been reported to be caused by non-specific reactions of the kits when the specimen contains a large number of substances such as viscous substances and human anti-mouse antibodies (HAMAs) [29].

Moreover, it was not possible to determine if the false-positive results were caused by cross-reactivity with B. parapertussis and B. holmesii in the present study (Supplementary Table S2). Even if B. parapertussis 
or $B$. holmesii causes the infection, the treatment is the same as for $B$. pertussis infection. The doctor's diagnosis also suggested false-positive results with the ICkit. Therefore, we must remember that the ICkit may show false-positive results if specimens contain viscous substances, HAMAs, $B$. parapertussis, or $B$. holmesii.

Essentially, the ICkit can be positive in the presence of the antigen regardless of age. In the present study, the ICkit detected the antigen in both children and adults similarly to rPCR (Fig. 3). However, the ICkit may produce false-negative results due to the disappearance of the bacteria if time has passed since onset or if patients take antimicrobials. Therefore, caution is needed regarding the timing of ICkit use. The CDC recommends the implementation of culture within two weeks and PCR within four weeks [10]. The period during which the ICkit detected the antigen was up to the 25th day, the same as rPCR in the clinical study (Fig. 4). Nakamura et al. reported that colonization tended to be less in adults than in children [30]. In addition, adults often have mild symptoms with atypical cough for pertussis and may delay seeking medical attention [31]. Therefore, we should ask for epidemiological information such as household infections and outbreaks to use the ICkit early after onset. In the present study, all ICkit-positive cases in adults were tested within two weeks of contact with infected cases. When the antigen has already disappeared over time, an antibody test should be an option for diagnosis [32, 33]. Although the results of the present study were limited to three positive cases of rPCR in adults, further consideration of how to use the ICkit in adults is needed.

The characteristics of the ICkit's rapidity and convenience suggest three major points. First, the ICkit would prevent infected people from getting more severe illness. The ICkit would contribute to early diagnosis and increase the opportunities for administering a macrolide, which is the first-line agent for pertussis, in the early stage [34,35]. If macrolides can suppress bacterial growth during the catarrhal phase, they can suppress the production of toxins that exacerbate respiratory symptoms. Based only on clinical symptoms, it is often difficult to distinguish B. pertussis infection from viral and other bacterial respiratory tract infections without any characteristic symptoms, which delays the administration of macrolides [1]. Both clinical symptoms and test results are important to identify and treat pertussis in the early stage. Second, the ICkit would help in antimicrobial stewardship [36, 37]. In the present study, about $30 \%$ of cases received antimicrobials before laboratory confirmation. The shorter turnaround time with the ICkit enables doctors to check the test results on the spot and to decide whether patients need to receive antimicrobials within the first consultation. If the test results rule out pertussis, and a viral respiratory tract infection is suspected, we will refrain from unnecessary antibiotics. Due to the side effect of hypertrophic pyloric stenosis, we must avoid unnecessary macrolides in infants without testing [38, 39]. Antimicrobial stewardship is essential, because antimicrobial-resistant strains of $B$. pertussis have already been identified [40-42]. Third, the ICkit would contribute to preventing spread of the infection. Delay in diagnosis and treatment increases the risk of transmission and further expands large-scale outbreaks $[11,43]$. The ICkit will be useful in environments where culture and nucleic acid tests are not actively available or in urgent situations such as an outbreak. In such situations, the easy accessibility of the ICkit would be beneficial for checking whether people around the infected person are infected. In institutions that have difficulty performing tests, the ICkit would make it possible to identify $B$. pertussis 
that has been missed so far. The potential benefits of the ICkit would be maximized in low-resource countries with many infected people due to low vaccine coverage and where conventional testing for pertussis is difficult due to immature infrastructure $[44,45]$.

In conclusion, a new, rapid immunochromatographic antigen kit for $B$. pertussis was developed. Its procedure is simpler and faster than conventional tests, so that its result is available at the first visit of potentially infected people. Furthermore, the ICkit is a POCT that does not require any equipment and is available in any clinical setting. The dilution sensitivity test and the clinical study demonstrated that the ICkit could detect the B. pertussis antigen. The clinical performance of the ICkit was well correlated with the rPCR results and was demonstrated within the same time from cough onset as rPCR. However, to correctly interpret the ICkit's results, we must use the ICkit with a complete understanding of the ICkit's precautions, such as cross-reactivity, the method for collecting the specimen and, the timing of use. Easy and rapid detection of $B$. pertussis by the ICkit would help diagnose pertussis more rapidly and efficiently.

\section{Methods}

\section{Dilution sensitivity test.}

The dilution sensitivity test was conducted at Asahi Kasei Pharma. The test used four Bordetella strains, including BAA-589 (ATCC), 8467 (ATCC), 9797 (ATCC), and 9340 (ATCC). These strains were grown in Bordetella CFDN agar plates (Nikken Bio Medical Laboratory Inc., Kyoto, Japan) for 4 to 7 days at $37^{\circ} \mathrm{C}$ to a concentration of $1.0 \times 10^{9}$ colony forming units $/ \mathrm{mL}(\mathrm{CFU} / \mathrm{mL})$. These cultured strains were then suspended in phosphate-buffered saline (PBS) and used to investigate the detection limit of the ICkit. Samples applied to the ICkit were prepared from bacterial suspensions, reagent $A$, and reagent $B$. The test was performed at concentrations of $0.4 \times 10^{4}, 2.2 \times 10^{4}, 8.8 \times 10^{4}$, and $44 \times 10^{4} \mathrm{CFU} / \mathrm{mL}$, respectively.

\section{Clinical study.}

This prospective clinical study was conducted from October 2016 to September 2019 in 11 institutions in Japan. This study was approved by the ethics committees of Asahi Kasei Pharma, Tokyo Metropolitan Children's Medical Center, Konan Kosei Hospital, Miyazaki Prefectural Miyazaki Hospital, National Hospital Organization Fukuoka National Hospital, National Center for Child Health and Development, Yokohama Minoru Clinic, Sapporo Medical Association, and Aichi Children's Health and Medical Center. All procedures were performed in accordance with the Declaration of Helsinki and its amendments. All patients or acceptable representatives provided written, informed consent. The contents of the clinical study are published on UMIN-CTR (UMIN000041828). The subjects were patients suspected of being infected with $B$. pertussis. Patients who matched either of the following were enrolled and specimens taken: 1. patients who had a cough and presented with one or more characteristic symptoms of paroxysms, whooping, post-tussive vomiting, or apnea with or without cyanosis; or 2. patients who had a possibility of contact with an infected person. Patients from whom nasopharyngeal swab specimens were not available or who were judged by doctors to be inappropriate for this study were excluded. In 
every institution, two swab specimens were taken per patient by rubbing the posterior nasopharynx. One swab specimen was immediately used for the ICkit measurement at each institution. The residual specimen of the ICkit measurement was used for the rPCR measurement at Kawasaki Medical School. The other swab specimen was immediately used for culture at each institution.

\section{Immunochromatographic antigen kit (ICkit).}

The ICkit was approved as Ribotest ${ }^{\circledR}$ Pertussis (Asahi Kasei Pharma, Tokyo, Japan) in Japan for the purpose of detecting $B$. pertussis antigens in a nasopharyngeal swab specimen. Figure 1 shows the structure and principle of the ICkit. The procedure of the ICkit is performed by following the steps below.

- Take a postnasal specimen with a swab.

- Elute the specimen into reagent $A$ in a tube.

- Drop reagent $B$ into the tube and mix, with inversion of the extraction tube closed by a cap.

- Drop the sample onto the specimen dropping area of the test plate.

- Determine the result based on the presence or absence of a line in the determination part of the test plate in $15 \mathrm{~min}$.

The cross-reactivity test confirmed that the ICkit has cross-reactivity with Bordetella parapertussis and Bordetella holmesii (Supplementary Table S2).

\section{Real-time polymerase chain reaction (IPCR).}

rPCR targeting IS 481 was performed using the previously described method with some modifications [46]. In total, $50 \mu \mathrm{L}$ of the diluted test sample were used for DNA extraction, and total DNA was extracted using QIAamp DNA Micro Kit (Qiagen, Hilden, Germany). DNA amplification was performed using the CFX96TM Real-Time System (Bio-Rad, Hercules, CA, USA) and SsoFast Probes Supermix (Bio-Rad). In this study, IS 481 plasmid DNA was used as the internal control. A standard curve was also generated with 10 -fold serial dilutions of IS 481 plasmid DNA from $1.0 \times 10^{5}$ copies to $1.0 \times 10^{2}$ copies.

\section{Culture.}

B. pertussis was cultivated following the usual flow at each institution. The culture medium for $B$. pertussis was charcoal agar medium (Oxoid Ltd., Hampshire, UK) at Konan Kosei Hospital, cyclodextrin pyruvate solid medium at Miyazaki Hospital, and Bordetella CFDN agar medium (Nikken Bio Medical Laboratory Inc.) at the other institutions. Finally, B. pertussis was identified by an agglutination test.

\section{Statistical analysis.}

Statistical analysis was performed using Analyse-it version 5.66 (Analyse-it Software, Ltd., Leeds, UK).

\section{Declarations}


Acknowledgements

The clinical study was funded and supported by Asahi Kasei Pharma.

\section{Author Contributions}

All authors read and approved the final manuscript. C.A. performed experiments. K.Ok., K.Ou., and T.H. conceived and designed the clinical study. Y.H., N.N., S.I., H.N., C.M., I.M., N.T., T.M., K.I., S.H., K.N., and H.T. recruited and collected clinical data. K.Ok. and T.H. analyzed data and drafted the manuscript.

Competing interests: Funding for the clinical study was provided to the institutions of Y.H., N.N., S.I., H.N., C.M., I.M., N.T., T.M., K.I., S.H., K.N., and H.T. by Asahi Kasei Pharma. T.H. and C.A. are employees of Asahi Kasei Pharma. K.Ok. has received consultancy fees from Asahi Kasei Pharma. K.Ou. has received lecture fees from Asahi Kasei Pharma.

\section{Data Availability Statement.}

The datasets generated during and/or analyzed during the current study are available from the corresponding author on reasonable request.

\section{References}

1. Kilgore, P. E., Salim, A. M., Zervos, M. J. \& Schmitt, H. Pertussis: microbiology, disease, treatment, and prevention. Clin Microbiol Rev. 29. 449-486 (2016).

2. World Health Organization. Global Health Observatory data repository, By category, Vaccinepreventable communicable diseases, Pertussis, Reported cases by country. https://apps.who.int/gho/data/node.main.WHS3_43?lang=en. Accessed July 2021.

3. Surridge, J., Segedin, E. R. \& Grant, C. C. Pertussis requiring intensive care. Arch Dis Child. 92. $970-$ 975 (2007).

4. Winter, K. et al. Risk factors associated with infant deaths from pertussis: a case-control study. Clin Infect Dis. 61. 1099-1106 (2015).

5. Yeung, K. H. T., Duclos, P., Nelson, E. A. S. \& Hutubessy, R. C. W. An update of the global burden of pertussis in children younger than 5 years: a modelling study. Lancet Infect Dis.17. 974-980 (2017).

6. Hewlett, E. L. \& Edwards, K. M. Clinical practice. Pertussis - Not Just for Kids. N Engl J Med. 352. 1215-1222 (2005).

7. von König, C. H. W., Halperin, S., Riffelmann, M. \& Guiso, N. Pertussis of adults and infants. Lancet Infect Dis. 2. 744-50 (2002).

8. Wilkinson, K., Righolt, C. H., Elliott, L. J., Fanella, S. \& Mahmud, S. M. Pertussis vaccine effectiveness and duration of protection-A systematic review and meta-analysis. Vaccine. 39. 3120-3130 (2021).

9. World Health Organization. Laboratory manual for the diagnosis of whooping cough caused by Bordetella pertussis/ Bordetella parapertussis (2014). 
https://www.who.int/publications/i/item/laboratory-manual-for-the-diagnosis-of-whooping-coughcaused-by-bordetella-pertussis-bordetella-parapertussis.-update-2014. Accessed July 2021.

10. Center for Disease Control and Prevention. The Pink Book: Course Textbook-13th Edition, Chapter 16: Pertussis (2015). https://www.cdc.gov/vaccines/pubs/pinkbook/chapters.html. Accessed July 2021.

11. Goodenough, D., Thomas, E., Tuttle, J. \& Bednarczyk, R. A. Factors associated with time to appropriate treatment in pertussis cases in Georgia, 2009 to 2013. Antimicrob Agents Chemother. 60. 3051-3056 (2016).

12. Polinori, I. \& Esposito, S. Clinical Findings and Management of Pertussis. Adv Exp Med Biol. 1183. 151-160 (2019).

13. Ebell, M. H., Marchello, C. \& Callahan, M. Clinical Diagnosis of Bordetella Pertussis Infection: A Systematic Review. J Am Board Fam Med. 30. 308-319 (2017).

14. Gu, W. et al. Pathogen analysis of pertussis-like syndrome in children. BMC Infect Dis. 20. 353 (2020).

15. Nieves, D. J. \& Heininger, U. Bordetella pertussis. Microbiol Spectr. 4. (2016).

16. Liu, Y. et al. Etiological distribution of pertussis-like syndrome in 756 children in Chengdu. Transl Pediatr. 10. 984-989 (2021).

17. Mihara, Y. et al. Bordetella pertussis is a common pathogen in infants hospitalized for acute lower respiratory tract infection during the winter season. J Infect Chemother. 27. 497-502 (2021).

18. Greenberg, D. et al. Pertussis is under diagnosed in infants hospitalized with lower respiratory tract infection in the pediatric intensive care unit. Med Sci Monit. 13. 475-480 (2007).

19. van der Zee, A., Schellekens, J. F. P. \& Mooi, F. R. Laboratory Diagnosis of Pertussis. Clin Microbiol Rev. 28. 1005-1026 (2015).

20. Bocharov, E. V. et al. From Structure and Dynamics of Protein L7/L12 to Molecular Switching in Ribosome. J Biol Chem. 279. 17697-17706 (2004).

21. Lucer, C. A. \& Wong, K. P. Conformational stability of ribosomal protein L7/L12: effects of pH, temperature, and guanidinium chloride. Biochemistry. 19. 176-183 (1980).

22. Kolberg. J., Høiby, E. A., Lopez, R. \& Sletten, K. Monoclonal antibodies against Streptococcus pneumoniae detect epitopes on eubacterial ribosomal proteins L7/L12 and on streptococcal elongation factor Ts. Microbiology. 143. 55-61 (1997).

23. Sawa, T. et al. Diagnostic usefulness of ribosomal protein $L 7 / L 12$ for pneumococcal pneumonia in a mouse model. J Clin Microbiol. 51. 70-76 (2013).

24. Sano, G. et al. Characterization and evaluation of a novel immunochromatographic assay for pharyngeal Mycoplasma pneumoniae ribosomal protein L7/L12 antigens. J Med Microbiol. 65. 1105-1110 (2016).

25. Ito, A. et al. Evaluation of a novel urinary antigen test kit for diagnosing Legionella pneumonia. Int $J$ Infect Dis. 103. $42-47$ (2021). 
26. Dragsted, D. M., Dohn, B., Madsen, J. \& Jensen, J. S. Comparison of culture and PCR for detection of Bordetella pertussis and Bordetella parapertussis under routine laboratory conditions. J Med Microbiol. 53. 749-754 (2004).

27. Ding, Y., Wang, Q., Li, D., Yao, K. \& Wang, T. Abundance of the nasopharyngeal microbiome effects pertussis diagnosis and explains the sensitivity difference between bacterial culture and real-time PCR. Eur J Clin Microbiol Infect Dis. 39. 501-507 (2020).

28. Centers for Disease Control and Prevention. Pertussis Home, Clinicians, Diagnostic Testing, Specimen collection (2019). https://www.cdc.gov/pertussis/clinical/diagnostic-testing/specimencollection.html. Accessed July 2021.

29. Hsieh, H. V., Dantzler, J. L. \& Weigl, B. H. Analytical Tools to Improve Optimization Procedures for Lateral Flow Assays. Diagnostics (Basel). 7.29 (2017).

30. Nakamura, Y. et al. Marked difference between adults and children in Bordetella pertussis DNA load in nasopharyngeal swabs. Clin Microbiol Infect. 17. 365-370 (2011).

31. Miyashita, N. et al. Diagnostic value of symptoms and laboratory data for pertussis in adolescent and adult patients. BMC Infect Dis. 13.129 (2013).

32. Weinberger, R. et al. Long-lasting cough in an adult German population: incidence, symptoms, and related pathogens. Eur J Clin Microbiol Infect Dis. 37. 665-672 (2018).

33. Lee, A. D. et al. Clinical evaluation and validation of laboratory methods for the diagnosis of Bordetella pertussis infection: Culture, polymerase chain reaction (PCR) and anti-pertussis toxin IgG serology (IgG-PT). PLoS One. 13. e0195979 (2018).

34. Carlsson, R. M., von Segebaden, K., Bergstrom, J., Kling, A. M. \& Nilsson, L. Surveillance of infant pertussis in Sweden 1998-2012; severity of disease in relation to the national vaccination programme. Euro Surveill. 20. 21032 (2015).

35. Winter, K. et al. Risk Factors Associated With Infant Deaths From Pertussis: A Case-Control Study. Clin Infect Dis. 61. 1099-1106 (2015).

36. Årdal, C. et al. International cooperation to improve access to and sustain effectiveness of antimicrobials. Lancet. 387. 296-307 (2016).

37. Arias, C. A. \& Murray, B. E. Antibiotic-resistant bugs in the 21 st century-a clinical super-challenge. $N$ Engl J Med. 360. 439-443 (2009).

38. Centers for Disease Control and Prevention. Hypertrophic pyloric stenosis in infants following pertussis prophylaxis with erythromycin-Knoxville, Tennessee, 1999. MMWR Morb Mortal Wkly Rep. 48. 1117-1120 (1999).

39. Eberly, M. D., Eide, M. B., Thompson, J. L. \& Nylund, C. M. Azithromycin in early infancy and pyloric stenosis. Pediatrics. 135. 483-488 (2015).

40. Yang, Y. et al. Variation in Bordetella pertussis Susceptibility to Erythromycin and Virulence-Related Genotype Changes in China (1970-2014). PLoS One. 10. e0138941 (2015). 
41. Wang, Z. et al. High prevalence of erythromycin-resistant Bordetella pertussis in Xi'an, China. Clin Microbiol Infect. 20. 825-830 (2014).

42. Ohtsuka, M. et al. Emergence of quinolone-resistant Bordetella pertussis in Japan. Antimicrob Agents Chemother. 53.3147-3149 (2009).

43. Terry, J. B. et al. A field study of household attack rates and the effectiveness of macrolide antibiotics in reducing household transmission of pertussis. Commun Dis Intell Q Rep. 39. E27-33 (2015).

44. Salminen, T., Knuutila, A., Barkoff, A., Mertsola, J. \& He, Q. A rapid lateral flow immunoassay for serological diagnosis of pertussis. Vaccine. 36. 1429-1434 (2018).

45. Posthuma-Trumpie, G. A., Korf, J. \& van Amerongen, A. Lateral flow (immuno) assay: its strengths, weaknesses, opportunities and threats. A literature survey. Anal Bioanal Chem. 393. 569-582 (2009).

46. Dragsted, D. M., Dohn, B., Madsen, J. \& Jensen, S. J. Comparison of culture and PCR for detection of Bordetella pertussis and Bordetella parapertussis under routine laboratory conditions. J Med Microbiol. 53. 749-754 (2004).

\section{Figures}
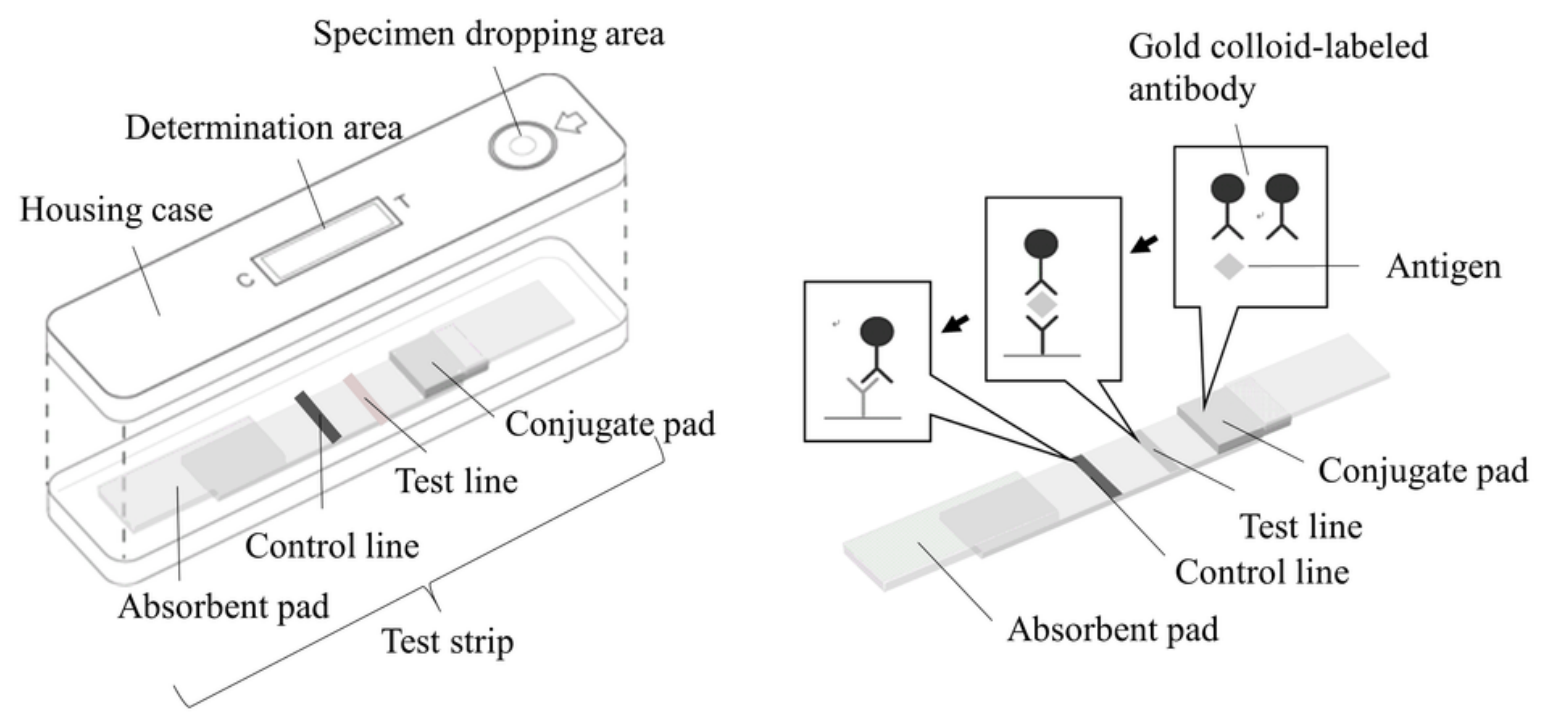

\section{Figure 1}

Structure and principle of the immunochromatographic antigen kit (ICkit). This product is a kit that detects Bordetella pertussis (B. pertussis) antigen in a nasopharyngeal swab specimen by 
immunochromatography. The kit includes reagent $A$, reagent $B$, and a test plate that consists of a test strip and a housing case. The specimen is applied to the test plate after pretreatment with reagent $A$ and reagent $B$. The specimen moves over the test strip by capillary action. When the specimen reaches the conjugate pad, the antigen causes an antigen-antibody reaction with the gold colloid-labeled monoclonal antibody (mouse) bound in the conjugate pad and forms an immunocomplex. This immunocomplex flows to the absorbent pad through the nitrocellulose membrane by capillary action. When reaching the test line, it is captured by the immobilized monoclonal antibody to form a sandwich immunocomplex. The sandwich immunocomplex results in a purplish-red line appearing at the determination part on the test plate. The presence or absence of this line can thus determine the presence or absence of the $B$. pertussis antigen. The control line appears as a purplish-red line of gold colloid-labeled monoclonal antibody when this antibody flows over the membrane and binds to a solid-phase anti-mouse immunoglobulin polyclonal antibody (rabbit), irrespective of the presence or absence of the B. pertussis antigen.

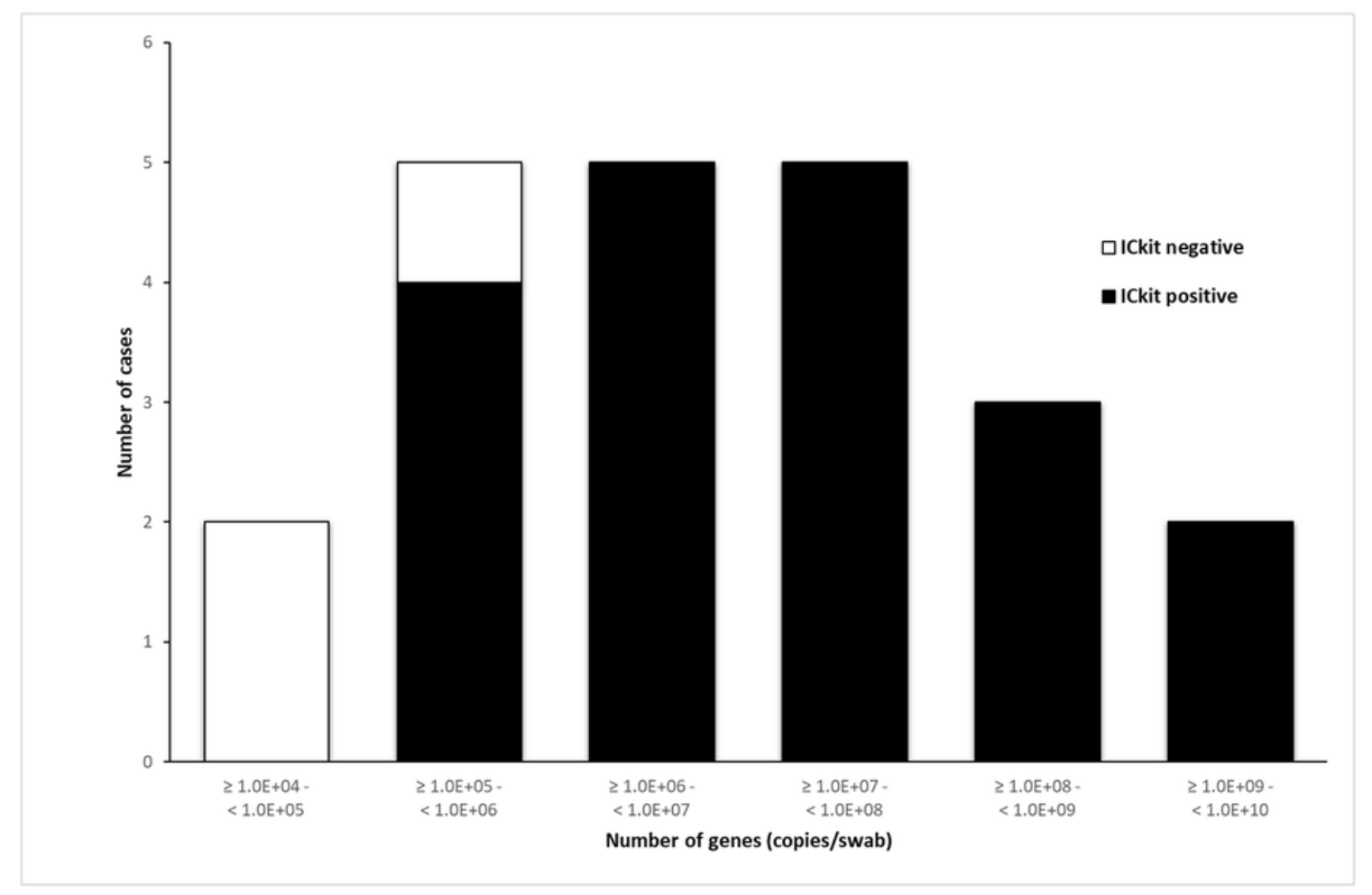

\section{Figure 2}

The relationship between the results of the ICkit and the number of IS481 genes by rPCR. A total of 22 positive cases were analyzed by rPCR. In the three negative cases, IS481 genes were detected by rPCR, but the ICkit was negative. ICkit: immunochromatographic antigen kit. rPCR: real-time polymerase chain reaction. 


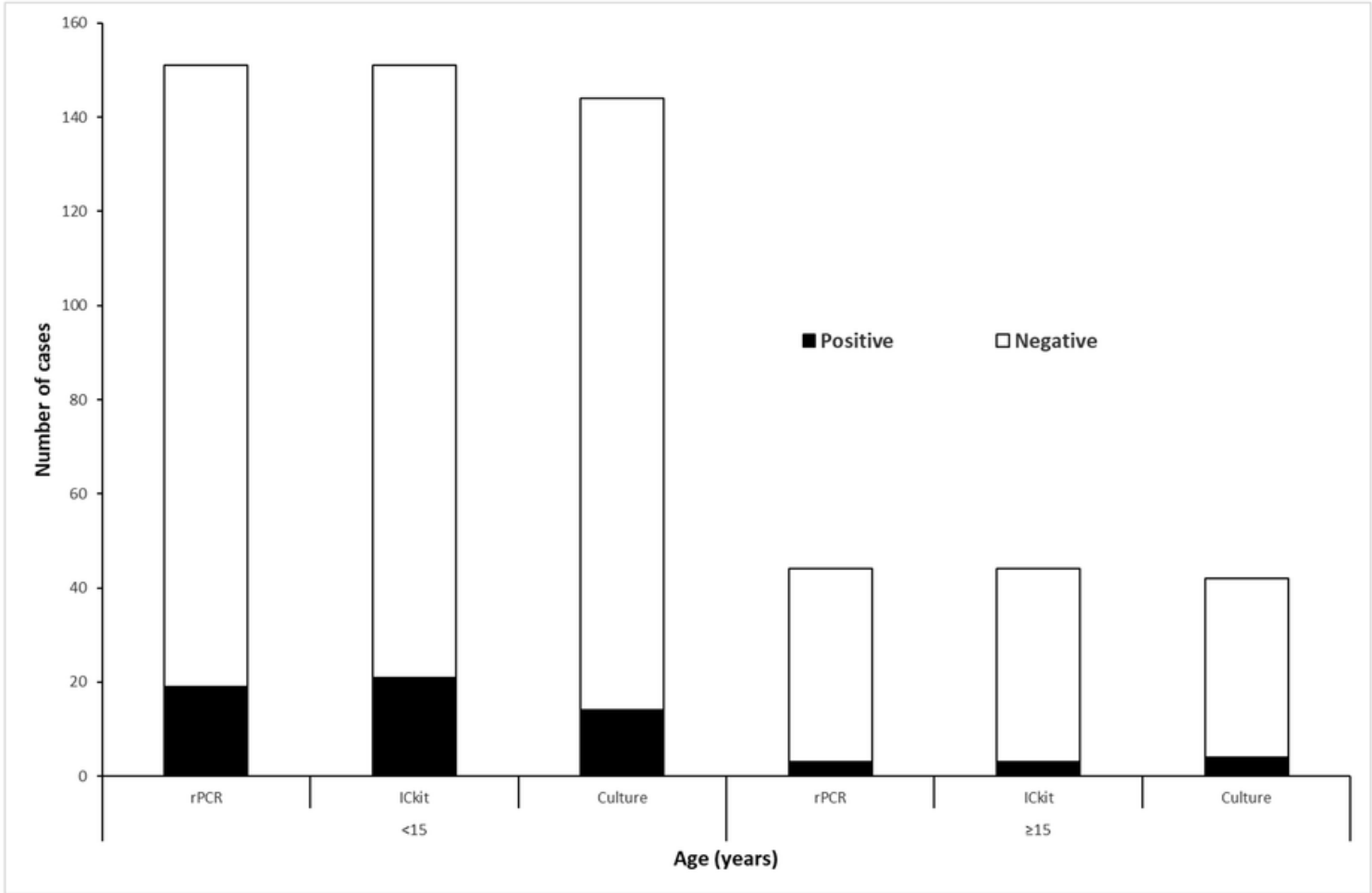

\section{Figure 3}

The results of rPCR, the ICkit, and culture by age groups. A total of 195 cases were analyzed. Nine cases were tested by the ICkit and rPCR but not tested by culture. ICkit: immunochromatographic antigen kit. rPCR: real-time polymerase chain reaction. 


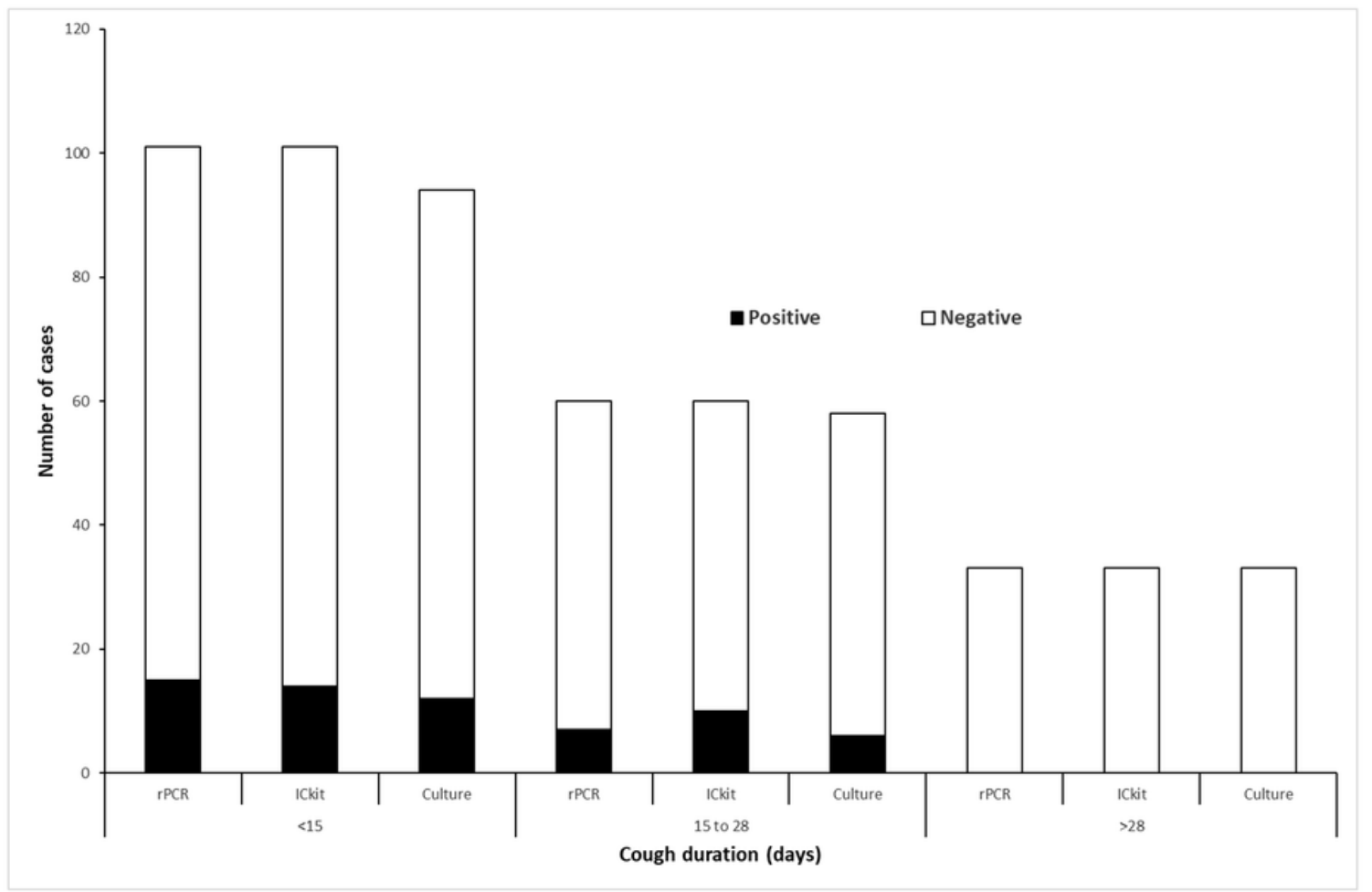

\section{Figure 4}

The results of rPCR, the ICkit, and culture by cough duration groups. A total of 194 cases with cough were analyzed. Of them, nine cases were tested by the ICkit and rPCR but not tested by culture. ICkit: immunochromatographic antigen kit. rPCR: real-time polymerase chain reaction.

\section{Supplementary Files}

This is a list of supplementary files associated with this preprint. Click to download.

- SupplmentaryInformation.docx 